Research Paper

\title{
Autoregulation of Inducible Nitric Oxide Synthase Expression by RNA Interference Provides Neuroprotection in Neonatal Rats
}

\author{
Zhi Wang ${ }^{* 1,2}$, Chenzhuo Feng ${ }^{*}{ }^{1}$, Huijuan Zhao ${ }^{1}$, Xiaoyan Ren ${ }^{1}$, Shuling Peng ${ }^{2}$, Zhiyi Zuo ${ }^{1 凶}$ \\ 1. Department of Anesthesiology, University of Virginia, Charlottesville, Virginia \\ 2. Department of Anesthesiology, Sun Yat-Sen Memorial Hospital, Sun Yat-Sen University, Guangzhou, Guangdong, China. \\ *ZW and CF contributed equally to this project. \\ \#Current address: Institute of Aging Research, University of Hangzhou Normal University, Hangzhou, Zhejiang, China
}

$\triangle$ Corresponding author: Dr. Zhiyi Zuo, Department of Anesthesiology, University of Virginia Health System, 1 Hospital Drive, PO Box 800710, Charlottesville, Virginia 22908-0710. Tel: 434-924-2283, Fax: 434-924-2105, e-mail: zz3c@virginia.edu

(c) 2015 Ivyspring International Publisher. Reproduction is permitted for personal, noncommercial use, provided that the article is in whole, unmodified, and properly cited. See http://ivyspring.com/terms for terms and conditions.

Received: 2014.08.29; Accepted: 2014.12.03; Published: 2015.02.15

\begin{abstract}
We have shown that autoregulation of gene expression by RNA interference is achievable in cell cultures. To determine whether this novel concept could be used to produce neuroprotection under in vivo condition, postnatal day (PND) 3 rats received intracerebroventricular injection of lentivirus that carried or did not carry code for short hairpin RNA (shRNA) of inducible nitric oxide synthase (iNOS). The expression of this shRNA was controlled by an iNOS promoter (piNOS-shRNA) or cytomegalovirus promoter ( $\mathrm{PCMV}$-shRNA). The rats were subjected to brain hypoxia-ischemia at PND7. Ischemic brain tissues had increased iNOS expression. This increase was attenuated by virus carrying piNOS-shRNA. Virus carrying PCMV-shRNA reduced iNOS to a level that was lower than control. Brain tissue loss and functional impairment after the hypoxia-ischemia were attenuated by the virus carrying piNOS-shRNA but not by PCMV-shRNA. Our results provide proof-of-concept evidence that autoregulation of iNOS expression by RNA interference induces neuroprotection in vivo and that appropriate regulation of gene expression is important.
\end{abstract}

Key words: autoregulation of gene expression; inducible nitric oxide synthase; neonate; neuroprotection; rats.

\section{Introduction}

RNA interference is a commonly used method to regulate gene expression [1]. This effect is often achieved by using small interfering RNA or short hairpin RNA (shRNA). Applying these small RNAs to cells under in vitro conditions is relatively easy but this application under in vivo conditions is difficult due to various issues, such as short life of these molecules and their inability to access target cells [1]. These issues can be solved by vector-mediated delivery. This form of delivery of shRNA holds a great therapeutic promise. The existing designs use pol III or tissue specific pol II promoters to control the expression of shRNA [2]. These designs allow robust shRNA production that is difficult to regulate and can saturate endogenous microRNA processing machinery to induce cell toxicity [3]. Introducing an inducible element to the promoters has been performed to achieve a better control of shRNA expression [4]. However, shRNA expression will be at its maximal level once the inducible element is activated. 
We proposed that optimal regulation of gene expression can be achieved by using the promoter of the gene to control the expression of shRNA for this gene product. Stimuli/factors that can increase the expression of a gene can also increase its shRNA expression via the same set of transcription factors. This autoregulation may keep the gene expression in a range that is close to its level under control condition. This fine regulation of gene/protein expression may be desirable for many potentially harmful proteins. For example, overexpression of inducible nitric oxide synthase (iNOS, gene I.D.: NM_012611.3) in the ischemic brain tissues is harmful [5-7]. However, iNOS has many physiological functions [8] and may induce ischemic tolerance in the brain [9-11]. Thus, maintaining an appropriate level of iNOS in the ischemic brain tissues may be desirable for reducing cell death and injury.

We have shown that autoregulation of iNOS expression is achievable in cell cultures [12]. We hypothesize that this autoregulation can provide neuroprotection against ischemia. To test this hypothesis, we subjected postnatal day (PND) 7 rats to brain hypoxia-ischemia $(\mathrm{HI})$ after they had received lentivirus carrying code for shRNA of iNOS.

\section{Methods and Materials}

All experimental protocols were approved by the institutional Animal Care and Use Committee of the University of Virginia (Charlottesville, VA). All surgical and experimental procedures were carried out in accordance with the National Institutes of Health Guide for the Care and Use of Laboratory Animals (NIH publications number 80-23) revised in 2011.

\section{Preparation of viruses}

Three plasmids generated in our previous study [12] were used here. piNOS-N had an unrelated insert whose expression was controlled by the $3.2 \mathrm{~Kb}$ iNOS promoter. This insert had a length similar to that of the iNOS shRNA [12] pCMV-shRNA and piNOS-shRNA had an iNOS shRNA whose expression was under control of the cytomegalovirus (CMV) promoter or iNOS promoter, respectively (Fig. 1). The shRNA was shRNA3 reported in the previous study [12]. These plasmids were used to transfect the 293FT cells to produce lentivirus as instructed by the protocol from Invitrogen (Carlsbad, CA). The viruses were purified and concentrated by using Fast-Trap Lentivirus Purification and Concentration kit from Millipore (Billerica, MA). All viruses were titrated by using Lentiviral titer kit from MellGen Laboratories Inc. (Surrey, BC, Canada). The titer of each viral solution was about $10^{10}$ transducing units $/ \mathrm{ml}$ in phosphate buffered saline. The virus was then aliquoted and kept at $-80^{\circ} \mathrm{C}$ until used.

\section{Neonatal cerebral hypoxia/ischemia model}

Cerebral hypoxia-ischemia (HI) was performed as we described before [10]. Briefly, PND7 male and female Sprague-Dawley rats were anesthetized by isoflurane in $30 \% \mathrm{O}_{2}-70 \% \mathrm{~N}_{2}$. Their right common carotid arteries were permanently ligated with a double 7-0 surgical silk. The neonates were returned to their cages with the mothers for $3 \mathrm{~h}$ and then placed in a chamber containing humidified $8 \% \mathrm{O}_{2}-92 \% \mathrm{~N}_{2}$ for $2 \mathrm{~h}$ at $37^{\circ} \mathrm{C}$.

\section{Intracerebroventricular injection of viruses}

PND3 or PND1 rats were anesthetized by isoflurane. A 26 gauge needle attached to a $5-\mu 1 \mathrm{Ham}-$ ilton Glass Microliter Syringe (Hamilton, Reno, Nevada) was used for intracerebroventricular injection. The co-ordinates were $1-1.5 \mathrm{~mm}$ rostral to the neonatal coronary suture, $1.0 \mathrm{~mm}$ on the right side of the sagittal suture and $2.5 \mathrm{~mm}$ ventral from the surface of the skull. One microliter of viral solution or vehicle $(0.9 \% \mathrm{NaCl}, \mathrm{pH} 7.4)$ was injected into the right ventricle. The needle was kept in place for $30 \mathrm{~s}$ after cease of plunger movement to prevent backflow. The rats were then kept in a warmed container for $10 \mathrm{~min}$ until normal movement and general responsiveness were restored.
Fig. 1. Diagram of the construct containing shRNA for iNOS (gene I.D.: NM_0I26II.3). The expression of this shRNA is under control of an iNOS or CMV promoter. The shRNA is shRNA3 in our previous publication.[12] 


\section{Brain injury/loss quantification}

As we described before [9], rats were sacrificed under deep isoflurane anesthesia and their brains were removed at 7 days after the brain HI. The hindbrain was removed from the cerebral hemispheres and the two hemispheres were separated and weighed. The weight ratio of right to left hemispheres was calculated.

\section{Brain histopathology}

This was performed as we described previously [10]. Rats were sacrificed under deep isoflurane anesthesia and transcardially perfused with cold normal saline one month after the brain HI. Brains were harvested, fixed in $4 \%$ paraformaldehyde at $4^{\circ} \mathrm{C}$ for $24 \mathrm{~h}$, and then embedded in paraffin. Five-micrometerthick coronal sections at approximately $3.3 \mathrm{~mm}$ caudal to Bregma were obtained and subjected to Nissl staining. These sections were assessed by an observer blinded to the group assignment of the sections. The cerebral areas in each of the hemispheres were quantified by using National Institutes of Health Image 1.60 (NIH, Bethesda, MD, USA). The cerebral area ratio of the right hemisphere to the left hemisphere was calculated to reflect brain tissue loss in the right hemisphere after brain HI. The number of Nissl staining positive cells (neurons) in a high magnification field $\left(\times 400, \sim 0.2 \mathrm{~mm}^{2}\right)$ in the CA3 and CA1 regions was determined. Three determinations, each on different locations in these two brain regions, were performed, and averaged to yield a single number (neuronal density) for each brain region of individual rat.

\section{Barnes maze}

One week before the rats were euthanized for brain histopathology, they were evaluated by a Barnes maze as we described previously [13] with minor modifications. The test was run and evaluated by a person blinded to the group assignment of rats. Rats were placed in the middle of a circular platform with 20 equally spaced holes. One hole was linked to a dark chamber and is named target hole. Rats were encouraged to find this box by aversive noise $(85 \mathrm{~dB})$ and bright light $(200 \mathrm{~W})$ shed on the platform. The protocol included training sessions on 4 consecutive days and four training sessions on each day with a 15-min inter-session interval. Each session ended when the rat went into the target hole or after $3 \mathrm{~min}$ had elapsed. On fifth day, one trial was carried out to test the retention ability of rats. All trials were documented and the latency for the rat to enter the target hole was calculated by using the ANY-Maze tracking system.

\section{Motor coordination evaluation}

Motor coordination was assessed 4 days before the rats were euthanized for brain histopathology as we described before [14]. Rats were placed on a rotarod whose speed increased from 4 to $40 \mathrm{rpm}$ within $5 \mathrm{~min}$. Before the formal tests, all rats were trained for two consecutive days. The latency and speed of rat's falling off the rotarod were documented. The speed-latency index (latency in seconds $x$ speed in rpm) of each test was calculated. Each rat was examined for three times in the formal test. The mean index value of the three trials was utilized to reflect the motor coordination functions of each rat.

\section{Fear conditioning}

One day after the completion of Barnes maze, fear conditioning test was carried out as we described before [15] and evaluated by a person blinded to the group assignment of rats. Briefly, each rat was placed in a Plexiglas conditioning training chamber cleaned with $70 \%$ alcohol. After a 3-min baseline exploratory episode in the chamber, rats were given 3 tone $(2000$ $\mathrm{Hz}, 90 \mathrm{db}$ )-shock (1 mA, $2 \mathrm{~s}$ ) pairings with 1-min interval between each pairing in a relatively dark room. Each rat was placed again $24 \mathrm{~h}$ after the training session in the training chamber for a period of $8 \mathrm{~min}$ in the absence of tone and foot shock to evaluate their contextual fear conditioning. The amount of time with freezing behavior was recorded in an 8-s interval. Two hour later, the rat was put in another test chamber that had different context and smell from the first test chamber (this second chamber was wiped with $1 \%$ acetic acid) in a relatively light room. After a 3-min exploratory term in this new chamber, the tone then was turned on for 3 cycles, each cycle for $30 \mathrm{~s}$ followed by 1 -min inter-cycle interval ( $4.5 \mathrm{~min}$ in total). Freezing behavior during this period was recorded.

\section{Western blot analysis}

The right cerebrum was harvested $24 \mathrm{~h}$ after the brain $\mathrm{HI}$ or 4 days after the intracerebroventricular injection of viruses. The cerebral tissues were homogenized in RIPA buffer (Cat. No. 89901; Thermo Scientific, Worcester, MA) containing protease inhibitor cocktail (Sigma, St. Louis, MO). The homogenates were centrifuged at $13,000 \mathrm{~g}$ at $4^{\circ} \mathrm{C}$ for $15 \mathrm{~min}$. The supernatant was saved and its protein concentration was determined by Bradford assay.

Equal protein samples (30 $\mu \mathrm{g}$ per lane) were separated by $10 \%$ sodium dodecyl sulfate-polyacrylamide gels and then electrotransferred onto nitrocellulose membranes (Bio-Rad, Hercules, CA). After being blocked with Protein-Free T20 Blocking Buffer (Cat. No. 37573, Thermo Scientific, Waltham, MA), membranes were incubated with the 
following primary antibodies: anti-iNOS antibody (1:1000; Santa Cruz Biotechnology, Santa Cruz, CA), anti-neuronal nitric oxide synthase (nNOS) antibody (1:1000; Cell Signaling Technology, Beverly, MA), anti-interleukin (IL)-1 $\beta$ antibody (1:1000; Abcam, Cambridge, MA), anti-IL-6 antibody (1:1000; Abcam), and anti-glyceraldehyde 3-phosphate dehydrogenase (GAPDH) antibody (1:2000; Sigma). Appropriate secondary antibodies were used. Protein bands were visualized and quantified using G:Box equipped with gene tools analysis software (Syngene, Frederick, MD, USA). The densities of protein bands were normalized to those of GAPDH to control for errors in protein sample loading and transferring during Western blotting. The results in the groups with brain HI were then normalized to those of control rats.

\section{Immunofluorescence}

The staining was performed as we have described before [16]. Briefly, rats were sacrificed by deep isoflurane anesthesia and transcardially perfused with $4 \%$ paraformaldehyde at $24 \mathrm{~h}$ after the brain HI or 4 days after the intracerebroventricular injection of the virus carrying pCMV-shRNA. Brains were harvested, fixed in $4 \%$ paraformaldehyde at $4^{\circ} \mathrm{C}$ for $18 \mathrm{~h}$ and then embedded in paraffin. After deparaffinization and antigen retrieval, $5-\mu \mathrm{m}$ thick sections at approximately $2.1 \mathrm{~mm}$ caudal to Bregma were incubated with $5 \%$ normal donkey serum and $1 \%$ bovine serum albumin in Tris-buffered saline for $2 \mathrm{~h}$ at room temperature. To reveal the co-localization of iNOS and ionized calcium binding adapter molecule 1 (Iba-1), sections were incubated with rabbit polyclonal anti-iNOS antibody (1:200; Abcam) and goat polyclonal anti-Iba-1 antibody (1:200; Abcam) and then donkey anti-rabbit IgG antibody conjugated with Alexa Fluor 488 (1:200; Life Technologies, Grand Island, NY) and donkey anti-goat IgG antibody conjugated with Alexa Fluor 594 (1:200; Life Technologies). To evaluate the co-localization of iNOS with glial fibrillary acidic protein (GFAP), rabbit polyclonal anti-iNOS antibody (1:200; Abcam) and the mouse monoclonal anti-GFAP antibody (1:300; Millipore) and then the donkey anti-rabbit IgG antibody conjugated with Alexa Fluor 488 (1:200; Life Technologies) and donkey anti-mouse IgG antibody conjugated with Alexa Fluor 594 (1:200; Life Technologies) were used. The antibodies used to stain neuN were mouse monoclonal anti-neuN antibody (1:500; Millipore) and the donkey anti-mouse IgG antibody conjugated with Alexa Fluor 594 (1:200; Life Technologies). To determine the co-localization of green fluorescent protein (GFP) with Iba-1, GFAP, or neuN, the rabbit polyclonal anti-GFP antibody (1:500; Abcam) and the donkey anti-rabbit IgG antibody conjugated with Alexa Fluor
488 (1:200; Life Technologies) were applied. The sections were viewed under a Zeiss LSM 510 Meta confocal microscope (Jena, Germany). A negative control omitting the incubation with the primary antibody was included in all experiments.

To evaluate the transduction efficiency of the viral vector, the percentage of cells that are stained positively for GFP and Iba-1, GFP and GFAP, or GFP and neuN in the corresponding total Iba-1, GFAP of neuN positive cells was determined by an investigator blinded to the experimental conditions. These determinations were in five randomly selected viewing fields of cerebral cortex in each section. Twenty determinations (5 viewing fields $\mathrm{x} 4$ sections) from each rat were averaged to represent the transduction efficiency in each animal.

\section{Statistical Analysis}

Parametric results are presented as means \pm S.D. $(n \geq 6)$. Data during the training sessions of Barnes maze test were analyzed by two-way repeated measures analysis of variance. All other data were analyzed by one-way analysis of variance followed by Student-Newman-Keuls test after confirmation of normal distribution of the data or by Kruskal-Wallis analysis of variance on ranks followed by Student-Newman-Keuls test when the data are not normally distributed. A P $\leq 0.05$ was accepted as significant.

\section{Results}

\section{Virus carrying code for iNOS shRNA specifi- cally reduced iNOS expression}

To test whether in vivo iNOS expression was regulated by the autoregulation technique we developed in cell cultures, postnatal day (PND) 3 rats received intracerebroventricular injection of vehicle (normal saline) or viral solution. Four days later, they were subjected to right carotid arterial ligation and maintained in $8 \%$ oxygen for $2 \mathrm{~h}$ (brain $\mathrm{HI}$ ). The right cerebral hemisphere had increased iNOS expression at $24 \mathrm{~h}$ after the brain HI. This increase was not affected by virus carrying piNOS-N but was inhibited by virus carrying piNOS-shRNA or pCMV-shRNA. The virus carrying $\mathrm{pCMV}$-shRNA reduced iNOS to a level that was lower than the normal control level (Figs. 2A and 2B).

To determine whether a longer interval between the viral injection and brain $\mathrm{HI}$ would provide a more effective down-regulation of iNOS, PND1 rats received viral injection and then were subjected to brain $\mathrm{HI}$ at PND7. The pattern of changes in iNOS expression in different experimental groups was similar to that when virus was injected at PND3 (Figs. 2C and 
2D). The virus carrying piNOS-shRNA reduced $76 \%$ and $77 \%$ brain $\mathrm{HI}$-induced iNOS increase when the virus was injected at PND3 and PND1, respectively. It appears that there is no increase in the degree of iNOS down-regulation with the increase of 2 days between the viral injection and brain ischemia. Injection of virus at PND3 was used for the rest of experiments.

There was a detectable level of iNOS in the cerebral tissues of PND7 rats under control condition.
This iNOS expression was moderately reduced (by $\sim 32 \%$ ) by virus carrying piNOS-shRNA and was very significantly reduced (by $\sim 77 \%$ ) by virus carrying pCMV-shRNA (Fig. 2E and 2F). These results, along with the results of iNOS expression in the ischemic brain tissues, suggest that the virus carrying piNOS-shRNA may be a better approach to maintain iNOS expression to its normal control level than the virus carrying $\mathrm{pCMV}$-shRNA.
A

B

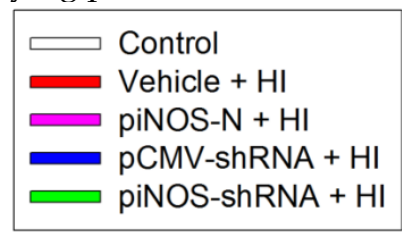

HI
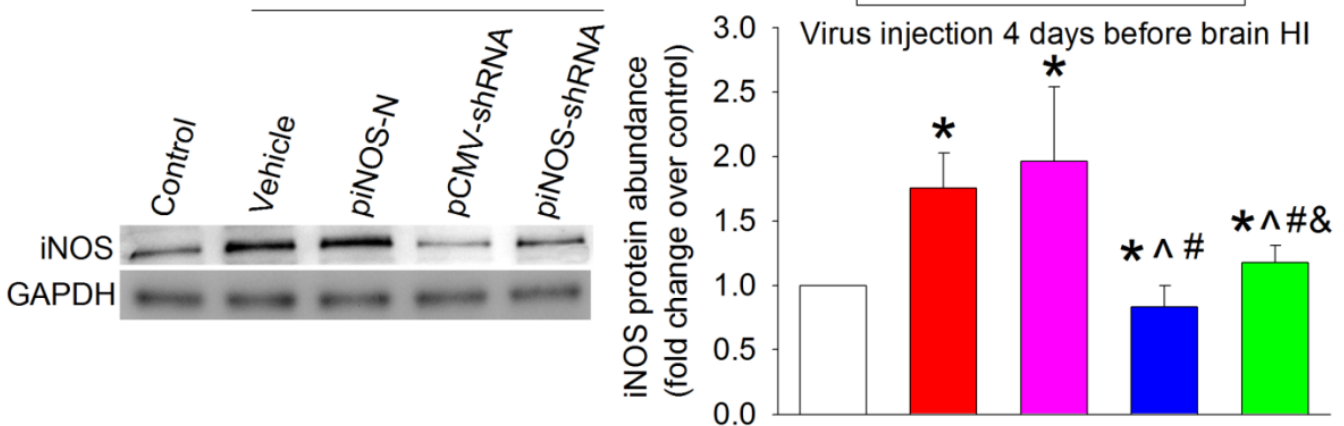

C

$\mathrm{HI}$

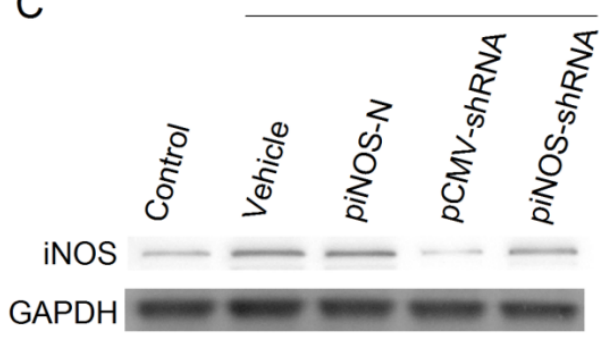

D
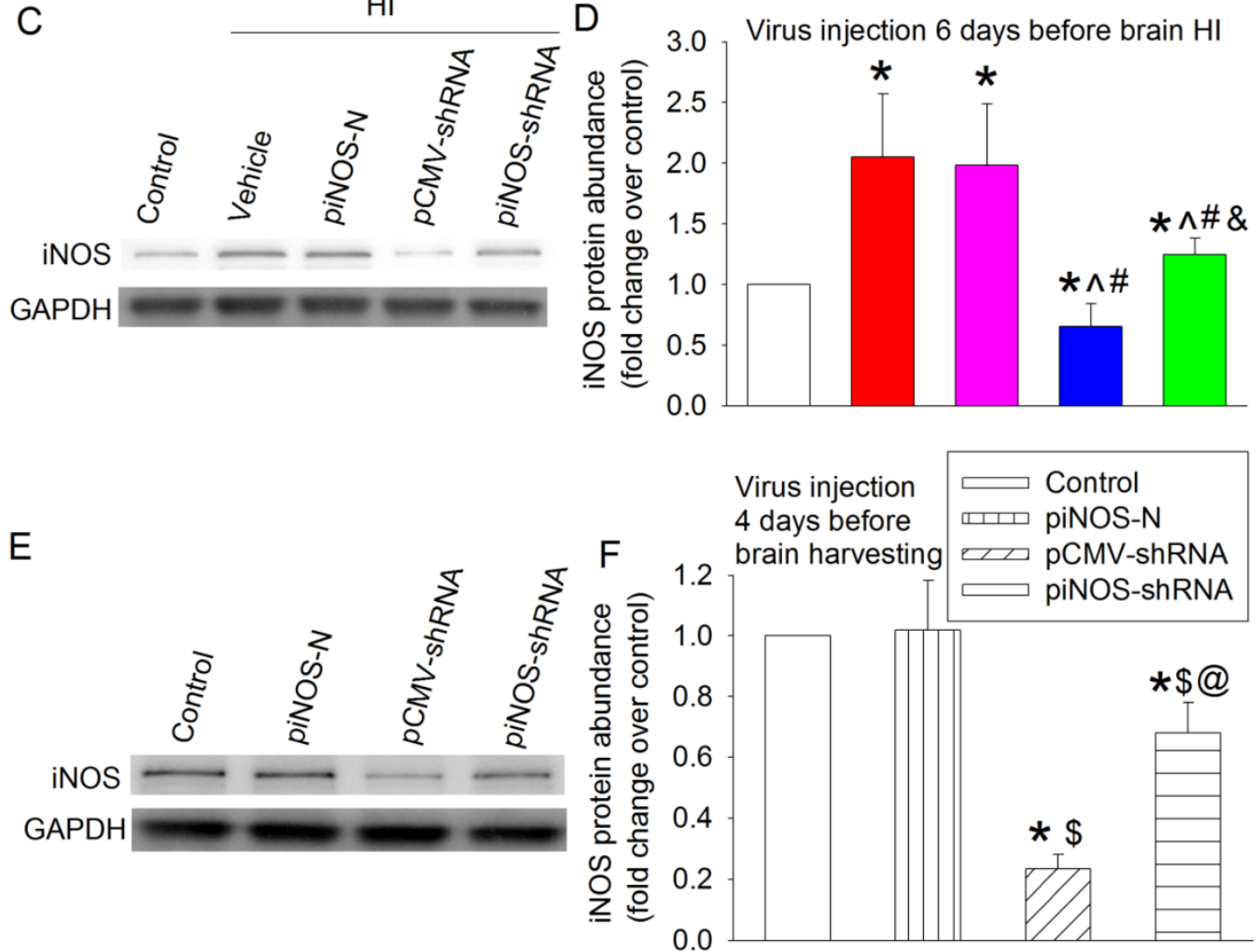

Fig. 2. Autoregulation of iNOS expression in the ischemic brain tissues. A to D: PND3 (panels A and B) or PND1 (panels C and D) rats received intracerebroventricular injection of lentivirus that carried or did not carry piNOS-shRNA. They were subjected to right brain HI at PND7. Right cerebral hemispheres were harvested at 24 $\mathrm{h}$ after the brain $\mathrm{HI}$ for Western blotting. Representative Western blotting images are shown in panels $\mathrm{A}$ and $\mathrm{C}$. The quantification results are presented in panels $\mathrm{B}$ and D. Results are mean \pm S.D. $(n=8)$. * P $<0.05$ compared with control, ^ $\mathrm{P}<0.05$ compared with vehicle plus brain $\mathrm{HI}$, \# $\mathrm{P}<0.05$ compared with piNOS-N plus brain $\mathrm{HI}$, \& $\mathrm{P}<0.05$ compared with $\mathrm{PCMV}$-shRNA plus brain $\mathrm{HI}$. E and $\mathrm{F}$ : PND3 rats received intracerebroventricular injection of lentivirus that carried or did not carry piNOS shRNA. Right cerebral hemispheres were harvested when they were PND7 for Western blotting. Representative Western blotting images are shown in panel E. The quantification results are presented in panel F. Results are mean \pm S.D. $(n=8)$. $* P<0.05$ compared with control, $\$ P<0.05$ compared with piNOS-N, @ P $<0.05$ compared with pCMV-shRNA. 
To determine the specificity of iNOS autoregulation, we measured nNOS level. The expression of nNOS in the ischemic brain tissues was reduced. This reduction was not affected by any viruses (Figs. 3A and $3 \mathrm{~B})$. These results, along with the results that virus carrying piNOS-shRNA reduced iNOS in the ischemic brain tissues and that virus carrying piNOS-N did not affect iNOS expression, suggest the specificity of the iNOS autoregulation by our technique. This suggestion is further strengthened by the results that IL-1 $\beta$ and IL- 6 were increased in the ischemic brain tissues and that these increases were not affected by virus carrying piNOS-shRNA or pCMV-shRNA (Figs.
$3 C-3 F)$.

Our viruses carried code for GFP [12]. To study the transduction efficiency, PND3 rats received intracerebroventricular injection of the virus carrying pCMV-shRNA. This lentivirus very effectively transduced neurons and microglia (> $94 \%$ of these cells were transduced) but poorly transduced astrocytes ( $\sim 3 \%$ transducing rate) in the cerebral cortex (Figs. 4A and 4B). Similar to this pattern, iNOS staining was co-localized very well with neuN, a neuronal marker, and Iba-1, a microglial marker. Some iNOS staining was co-localized with GFAP, an astrocytic marker (Fig. 4C).
A
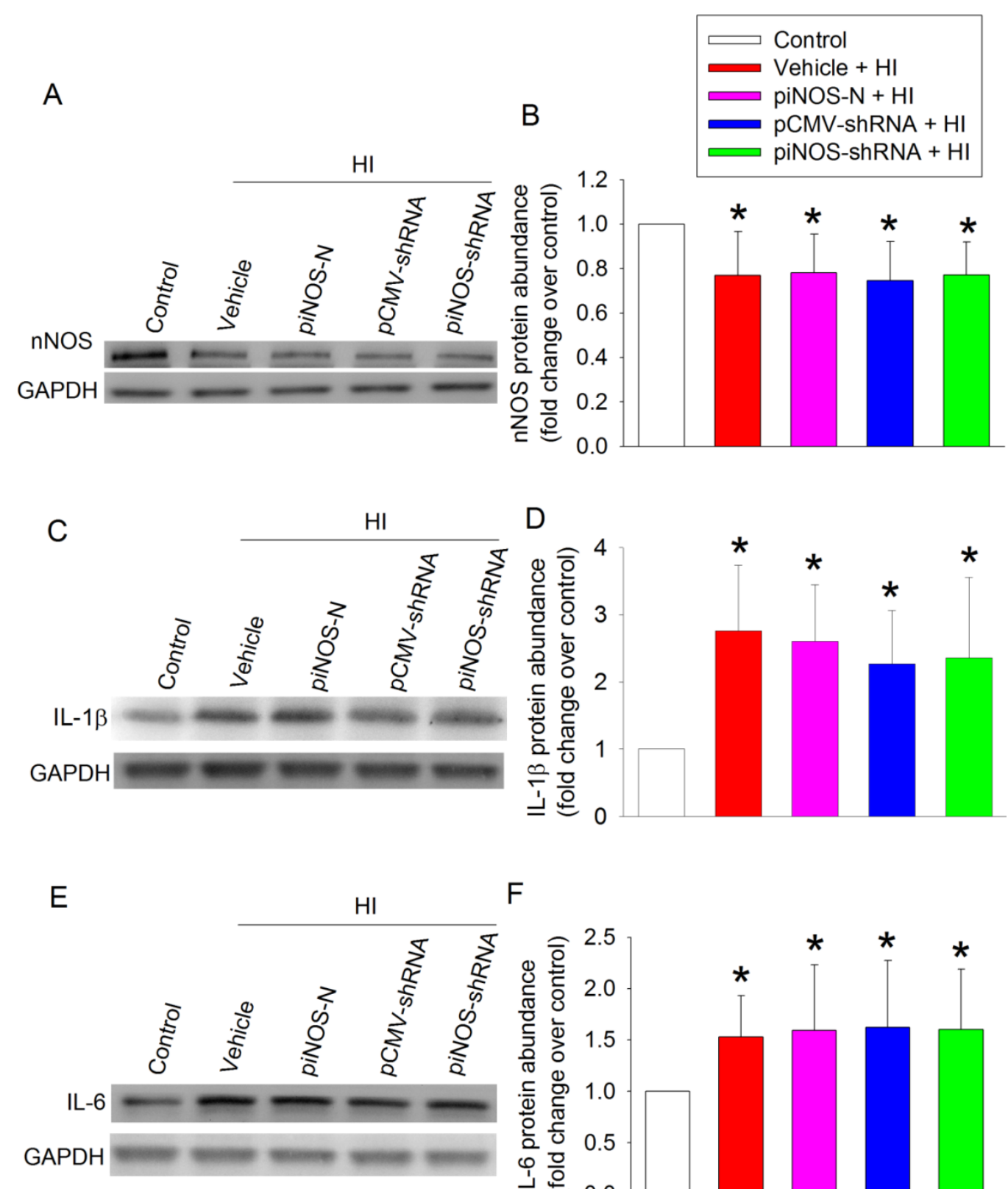

$\mathrm{F}$
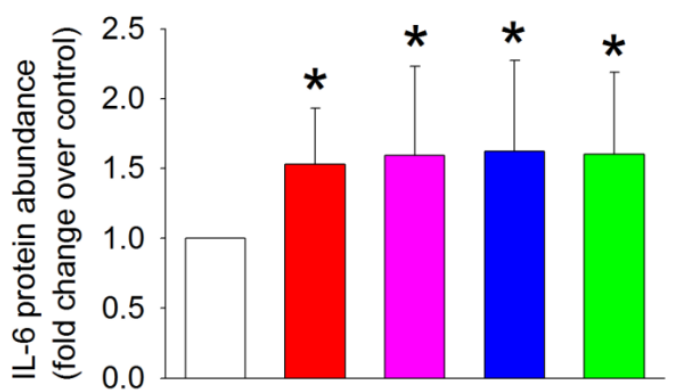

Fig. 3. No effect of iNOS shRNA on the expression of $n$ NOS and cytokines. PND3 rats received intracerebroventricular injection of lentivirus that carried or did not carry piNOS-shRNA. They were subjected to right brain $\mathrm{HI}$ at PND7. Right cerebral hemispheres were harvested at $24 \mathrm{~h}$ after the brain $\mathrm{HI}$ for Western blotting. A: Western blot image of $n$ NOS. B: quantification results of $n$ NOS. C: Western blot image of IL-1 $1 \beta$. D: quantification results of IL-1 $\beta$. E: Western blot image of IL-6. D: quantification results of IL-6. Results are mean \pm S.D. $(n=8)$. $* P<0.05$ compared with control. 


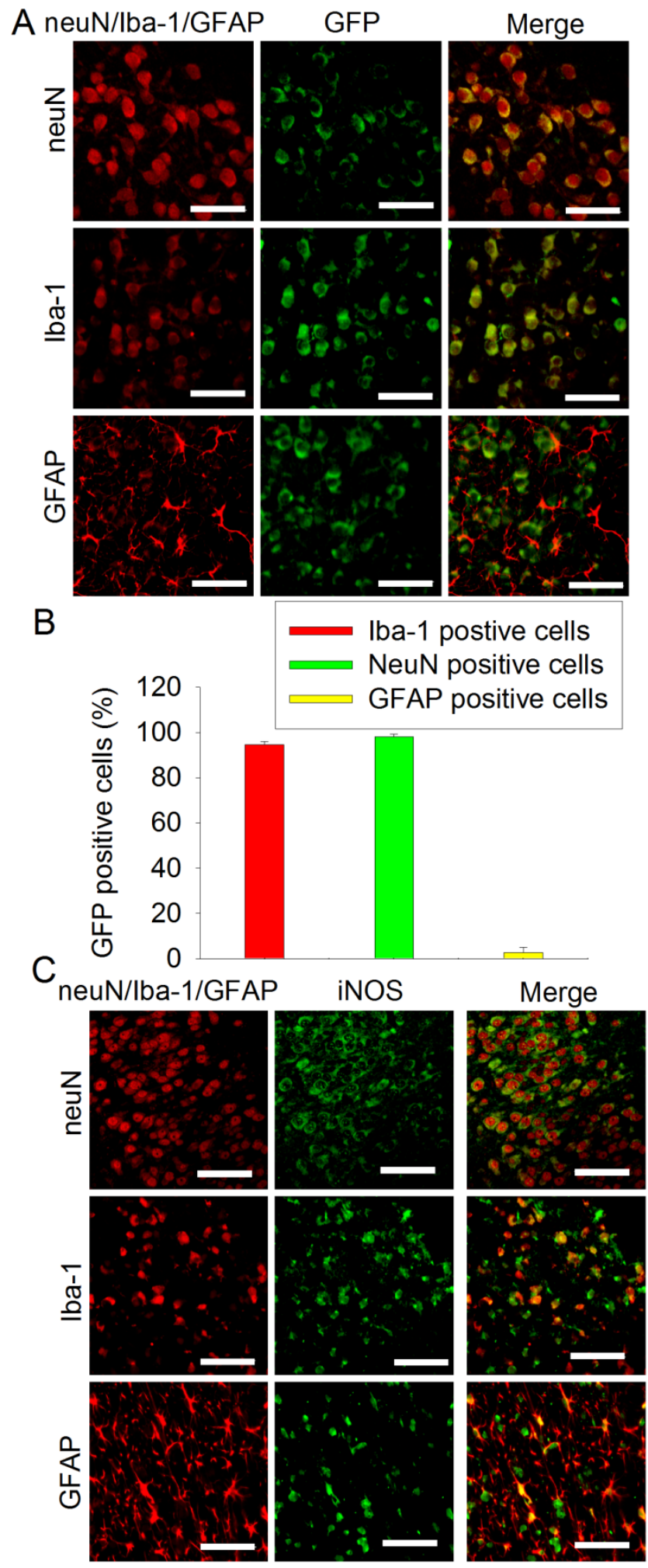

Fig. 4. Immunofluorescent staining of GFP and iNOS. A and B: PND3 rats received intracerebroventricular injection of lentivirus that carried or did not carry PCMV-shRNA. Their brain was harvested at PND7 for immunofluorescent staining. Representative images are shown in panel A. Quantification data of the percentage cells that were stained positively for neuN, Iba-1 or GFAP and also stained positively for GFP are presented in panel B. Results are mean \pm S.D. (n $=6$ ). C: PND7 rats without receiving any injection of virus were subjected to right brain $\mathrm{HI}$. Their brain was harvested $24 \mathrm{~h}$ after the brain $\mathrm{HI}$ for immunofluorescent staining. Scale bar in panels $A$ and $C$ equals to $50 \mu \mathrm{m}$.

\section{Autoregulation of iNOS expression provided neuroprotection}

To define the neuroprotection, we harvested the right and left cerebral hemispheres of rats and weighed them at 7 days after the brain HI. The weight ratio of right/left hemisphere was used to reflect brain tissue loss after brain $\mathrm{HI}$. The right brain $\mathrm{HI}$ induced significant tissue loss of the right cerebral hemisphere. This loss was attenuated by virus containing piNOS-shRNA but not by virus containing piNOS-N or pCMV-shRNA (Fig. 5).

To determine whether autoregulation of iNOS expression improved the long-term neurological outcome after brain ischemia, brain was harvested at one month after brain HI. Brain HI significantly damaged brain tissues and reduced the neuronal density in the CA1 and CA3 regions of the hippocampus. This brain injury was attenuated by virus carrying piNOS-shRNA but not by virus carrying pCMV-shRNA (Fig. 6). Consistent with these structural results, brain HI-impaired performance in rotarod, a method to assess motor coordination, was attenuated by virus carrying piNOS-shRNA but not by virus containing pCMV-shRNA (Fig. 7A).
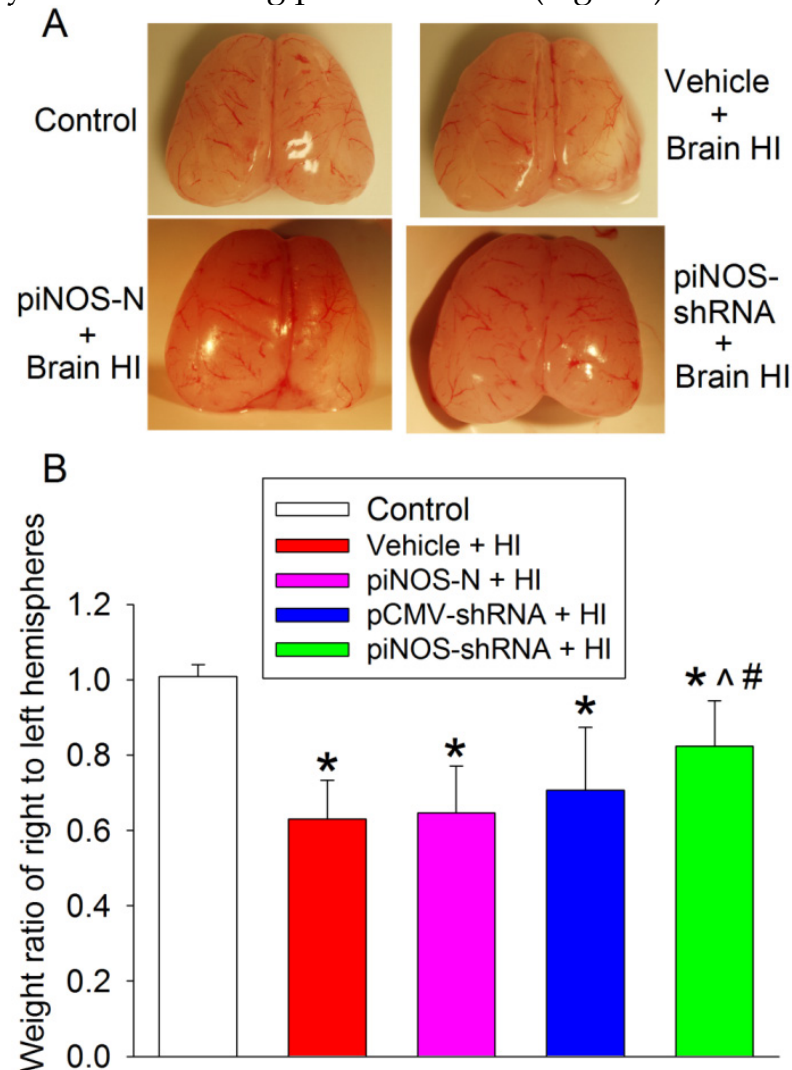

Fig. 5. Autoregulation of iNOS expression-induced neuroprotection. PND3 rats received intracerebroventricular injection of lentivirus that carried or did not carry piNOS-shRNA. They were subjected to right brain HI at PND7. Right and left cerebral hemispheres were harvested and weighed 7 days after the brain $\mathrm{HI}$. Results are mean \pm S.D. $(n=9-11) * P<0.05$ compared with control, $\wedge P<0.05$ compared with vehicle plus brain $\mathrm{HI}$, $\mathrm{P}<0.05$ compared with piNOS-N plus brain $\mathrm{HI}$. 
Barnes maze and fear conditioning tests were used to assess the learning and memory functions of rats. Control rats required decreased time to identify the target hole with increased training in the Barnes maze, a method to measure spatial working learning and memory [15]. This trend was no obvious in rats subjected to brain $\mathrm{HI}$ and reappeared in rats with brain $\mathrm{HI}$ plus receiving injection of the virus carrying piNOS-shRNA (Fig. 7B). Two-way repeated measures analysis of variance showed that brain $\mathrm{HI}$ was a significant factor for the learning and memory during the training sessions of Barnes maze test $[F(1,20)=47.207$, $\mathrm{P}<0.001]$. This effect was attenuated by the virus carrying piNOS-shRNA $[\mathrm{F}(1,22)=12.742, \mathrm{P}=0.002]$. Similar to the results during the training sessions, rats with brain $\mathrm{HI}$ took a longer time than control rats to identity the target hole one day after the training sessions in Barnes maze (Fig. 7C).

Rats with brain $\mathrm{HI}$ had less context-related freezing behavior than control rats. This decrease was attenuated by the virus carrying piNOS-shRNA (Fig. 7D). Similarly, rats with brain HI but not rats with $\mathrm{HI}$ plus receiving injection of the virus carrying piNOS-shRNA had less tone-related freezing behavior than control rats (Fig. 7E).

\section{Discussion}

Our results that virus carrying piNOS-shRNA reduced ischemic brain tissue loss and iNOS expression in the ischemic tissues provide proof-of-concept

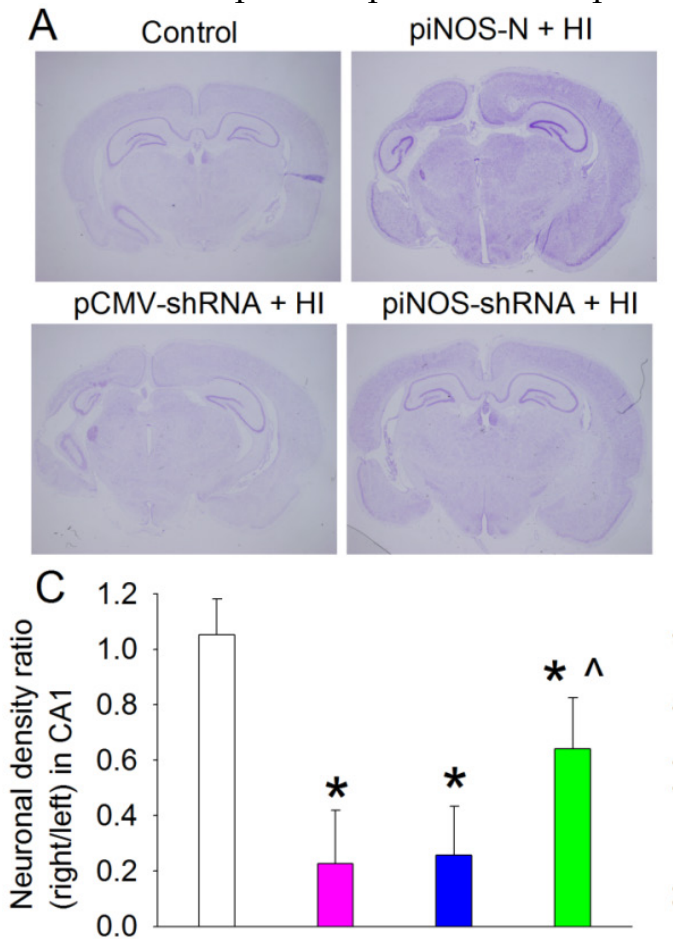

evidence that autoregulation of gene expression is achievable and can be very beneficial under in vivo conditions. In this study, the effects of iNOS autoregulation induced by virus carrying piNOS-shRNA should be the major mechanism for the neuroprotection caused by this virus because the damaging role of iNOS in the ischemic brain injury is well-established $[5-7,17]$. However, the role of nNOS in the ischemic brain injury after neonatal brain $\mathrm{HI}$ has also been shown [18]. Our results showed that virus carrying piNOS-shRNA did not affect nNOS expression in the ischemic brain tissues, suggesting that nNOS is not a target protein for the virus to induce neuroprotection. The reduced nNOS expression observed in the ischemic brain tissues in this study may be due to neuronal injury/death. Also, brain ischemia-induced neuroinflammation is an important neuropathological process for cell injury [13]. Inflammatory cytokines can induce iNOS expression [19]. Our results showed that IL-1 $\beta$ and IL-6 were increased in the ischemic brain tissues and that this increase was not affected by the virus carrying piNOS-shRNA, suggesting that affecting the proinflammatory cytokine expression may not be a mechanism for the virus carrying piNOS-shRNA to provide neuroprotection and to induce inhibition of iNOS expression in the ischemic brain tissues. Consistent with these findings, a previous study has shown that inhibition of both iNOS and nNOS improves neurological outcome but did not affect inflammatory cytokine production [20].

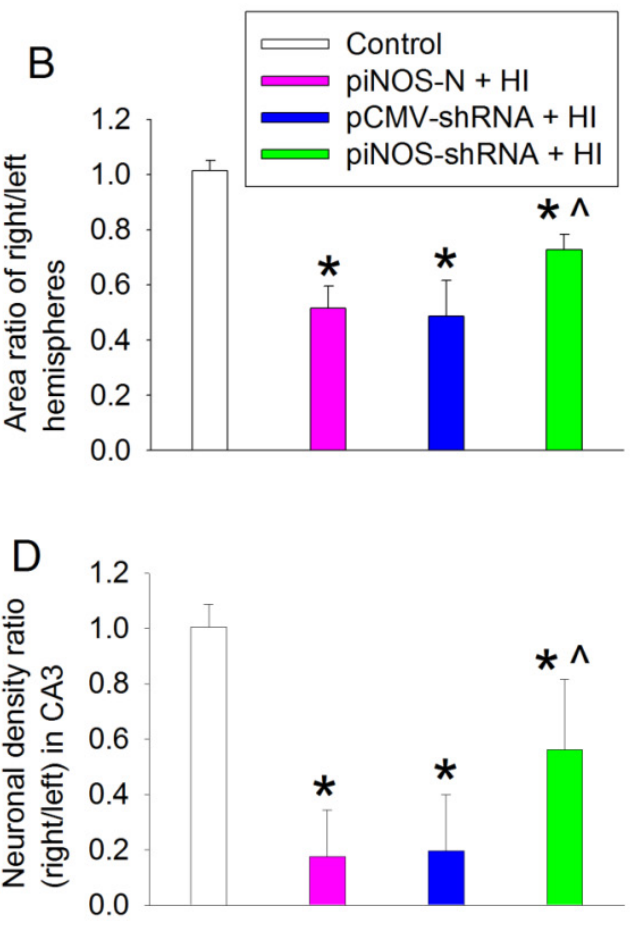

Fig. 6. Improvement of long-term brain structures by autoregulation of iNOS. PND3 rats received intracerebroventricular injection of lentivirus that carried or did not carry piNOS-shRNA. They were subjected to right brain $\mathrm{HI}$ at PND7. Their brain was harvested one month after the brain $\mathrm{HI}$ for Nissl staining. A: representative images of brain section at Bregma $-3.3 \mathrm{~mm}$. B: area ratio of right/left hemispheres. $\mathrm{C}$ and $\mathrm{D}$ : neuronal density ratio in the CA1 and CA3. Results are means \pm S.D. (n $=10-12) . * \mathrm{P}<0.05$ compared with control, $\wedge \mathrm{P}<0.05$ compared with piNOS-N plus brain $\mathrm{HI}$. 

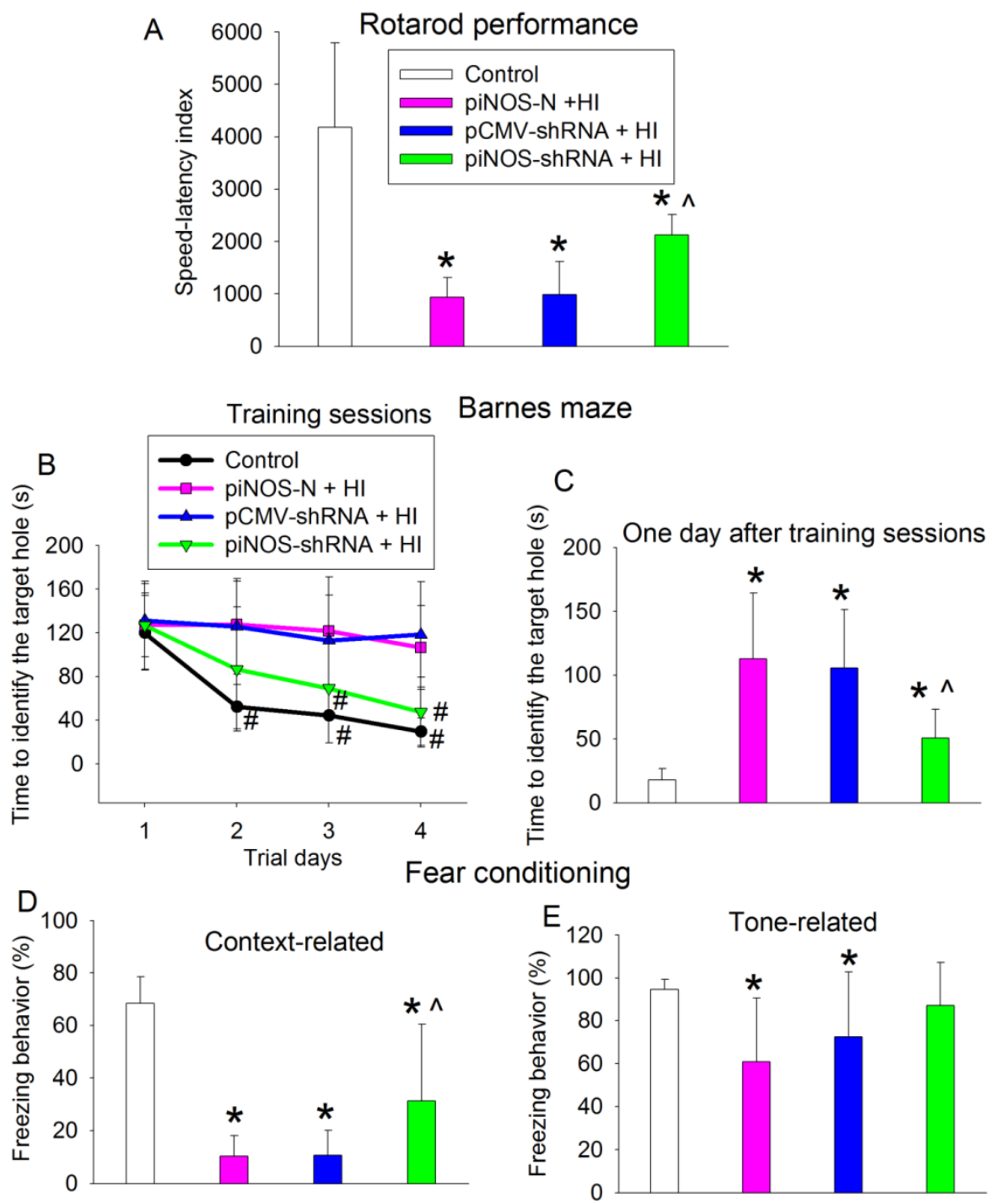

Fig. 7. Improvement of long-term neurological functions by autoregulation of iNOS. PND3 rats received intracerebroventricular injection of lentivirus that carried or did not carry piNOS-shRNA. They were subjected to right brain HI at PND7 and tested at least 23 days after brain HI. A: motor functions assessed by rotarod test. B and C: performance in Barnes maze. $D$ and E: performance in context- and tone-related fear conditioning. Results are means $\pm S . D$. $(n=10-12)$. $* P<0.05$ compared with control, ^ $\mathrm{P}<0.05$ compared with piNOS-N plus brain $\mathrm{HI}, \# \mathrm{P}<0.05$ compared with the corresponding values on day 1 .

Lentivirus can transduce dividing and non-dividing cells [21]. Our results showed that lentiviral vector very effectively transduced neurons and microglia. These cells are very active in producing proinflammatory cytokines and iNOS. However, lentivirus may not transduce astrocytes effectively as shown in our study. Improving the transduction of lentivirus to astrocytes has been achieved by gene engineering in a recent study [22].

The virus carrying piNOS-shRNA reduced $\mathrm{HI}$-induced brain tissue and neuronal loss assessed one month after the brain HI. Rats received virus carrying piNOS-shRNA also had better motor functions. Since context- and tone-related fear conditioning assesses hippocampus-dependent and hippocampus-independent learning and memory [23], virus carrying piNOS-shRNA also improved hippocam- pus-dependent and hippocampus-independent learning and memory after brain HI. These anatomical and functional results clearly showed that virus carrying piNOS-shRNA improved long-term neurological outcome after brain ischemia.

Our results clearly showed the damage of ipsilateral cerebral cortex and hippocampus after brain HI. Correspondingly, the performance of rats with brain $\mathrm{HI}$ on rotarod was poorer than that of control rats, indicating the injury of the motor strip. Spatial working learning and memory as assessed by Barnes maze was impaired in the rats after brain HI. These functions require cerebral cortex and hippocampus [24]. The context-related fear conditioning depends on hippocampus; whereas tone-related fear conditioning relies on thalamus, amygdale and cerebral cortex [23]. Thus, it is not surprising that the context- and 
tone-related fear conditioning was impaired after the brain HI.

An interesting finding is that the virus carrying pCMV-shRNA reduced iNOS expression in the ischemic brain tissues but did not provide significant neuroprotection. The exact mechanisms for this phenomenon are not known. However, iNOS has many physiological functions [8] and participates in induction of ischemic tolerance [9-11, 25] and neurogenesis after brain ischemia [26]. The reduction of iNOS expression to a level that was lower than that in control tissues may have affected these functions of iNOS. In addition, CMV promoter is very efficient. The abundant production of shRNA in cells infected by virus carrying pCMV-shRNA may have caused cell toxicity [3]. Nevertheless, the results from using this virus suggest the importance of proper control of gene expression.

We selected iNOS as the example to show the usefulness of autoregulation of gene expression in this study. It is clear that overexpression of iNOS in the ischemic brain tissues is harmful. The large amount of NO continuously produced from iNOS can react with superoxide to form peroxynitrite that then induces nitrosative and oxidative damage of proteins and lipids $[27,28]$. NO can also react with metal containing proteins to affect their functions [29]. These effects lead to cell injury after brain ischemia [5-7, 30]. However, a small amount of NO produced from iNOS is beneficial. It can activate cell survival signaling pathways, preserve cerebrovascular function and inhibit leukocyte adhesion [11, 31, 32]. iNOs is also involved in tissue repairing, such as neurogenesis, after brain ischemia [26, 33]. Thus, a proper level of iNOS expression is critical to reduce ischemic brain injury and improve neurological outcome after stroke.

The finding that autoregulation of iNOS provides neuroprotection may be very significant and have a broad implication. For example, iNOS and inflammatory cytokines play a role in inflammation [34]. Autoregulation of these proteins by RNA interference may hinder the pathological processes of many diseases involving inflammation.

\section{Acknowledgments}

This study was supported by a grant from the International Anesthesia Research Society (2007 Frontiers in Anesthesia Research Award to Z Zuo), Cleveland, Ohio, by grants (R01 GM065211 and R01 GM098308 to Z Zuo) from the National Institutes of Health, Bethesda, Maryland, by a Grant-in-Aid from the American Heart Association Mid-Atlantic Affiliate (10GRNT3900019 to Z Zuo), Baltimore, Maryland, and the Robert M. Epstein Professorship endowment, University of Virginia.

\section{Author contributions}

$\mathrm{ZW}, \mathrm{HZ}$ and $\mathrm{XR}$ performed the rat experiments. $\mathrm{CF}$ prepared the viruses. $\mathrm{ZW}$ and $\mathrm{CF}$ drafted the Methods and performed the initial data analysis. ZZ conceived the overall idea of the project, designed the experiments along with $\mathrm{CF}, \mathrm{ZW}$ and $\mathrm{SP}$, analyzed the data and wrote the paper.

\section{Conflict of interest}

None.

\section{References}

1. Agrawal N, Dasaradhi PV, Mohmmed A, Malhotra P, Bhatnagar RK, Mukherjee SK. RNA interference: biology, mechanism, and applications. Microbiol Mol Biol Rev 2003;67:657-85.

2. Xia XG, Zhou H, Xu Z. Promises and challenges in developing RNAi as a research tool and therapy for neurodegenerative diseases. Neurodegener Dis 2005;2:220-31

3. Grimm D, Streetz KL, Jopling CL, Storm TA, Pandey K, Davis CR, Marion P, Salazar F, Kay MA. Fatality in mice due to oversaturation of cellular microRNA/short hairpin RNA pathways. Nature 2006;441:537-41.

4. Xia XG, Zhou H, Xu Z. Multiple shRNAs expressed by an inducible pol II promoter can knock down the expression of multiple target genes. Biotechniques 2006;41:64-8.

5. Lipton P. Ischemic cell death in brain neurons. Physiol Rev 1999;79:1431-568.

6. Zhao $\mathrm{X}$, Ross ME, Iadecola C. L-Arginine increases ischemic injury in wild-type mice but not in iNOS-deficient mice. Brain Res 2003;966:308-11.

7. Garcia-Bonilla L, Moore JM, Racchumi G, Zhou P, Butler JM, Iadecola C, Anrather J. Inducible nitric oxide synthase in neutrophils and endothelium contributes to ischemic brain injury in mice. J Immunol 2014;193:2531-7.

8. Bogdan C. Nitric oxide and the immune response. Nat Immunol 2001;2:907-16.

9. Zhao $\mathrm{P}, \mathrm{Zuo} \mathrm{Z}$. Isoflurane preconditioning induces neuroprotection that is inducible nitric oxide synthase-dependent in the neonatal rats. Anesthesiology 2004;101:695-702.

10. Zhao P, Peng L, Li L, Xu X, Zuo Z. Isoflurane preconditioning improves long-term neurologic outcome after hypoxic-ischemic brain injury in neonatal rats. Anesthesiology 2007;107:963-70.

11. Kunz A, Park L, Abe T, Gallo EF, Anrather J, Zhou P, Iadecola C. Neurovascular protection by ischemic tolerance: role of nitric oxide and reactive oxygen species. J Neurosci 2007;27:7083-93.

12. Feng C, Cao L, Zuo Z. RNA interference-produced autoregulation of inducible nitric oxide synthase expression. FEBS Lett 2011;585:2488-92.

13. Li J, Sheng W, Feng C, Zuo Z. Pyrrolidine dithiocarbamate attenuates brain Abeta increase and improves long-term neurological outcome in rats after transient focal brain ischemia. Neurobiol Dis 2012;45:564-72.

14. Li L, Zuo Z. Isoflurane preconditioning improves short-term and long-term neurological outcome after focal brain ischemia in adult rats. Neurosci 2009;164:497-506.

15. Lin D, Zuo Z. Isoflurane induces hippocampal cell injury and cognitive impairments in adult rats. Neuropharmacology 2011;61:1354-9.

16. Wang $\mathrm{Z}$, Huang $\mathrm{W}, \mathrm{Zuo} \mathrm{Z}$. Perioperative aspirin improves neurological outcome after focal brain ischemia possibly via inhibition of Notch 1 in rat. J Neuroinflammation 2014;11:56.

17. Iadecola C, Zhang F, Casey R, Nagayama M, Ross ME. Delayed reduction of ischemic brain injury and neurological deficits in mice lacking the inducible nitric oxide synthase gene. J Neurosci 1997;17:9157-64.

18. Ferriero DM, Holtzman DM, Black SM, Sheldon RA. Neonatal mice lacking neuronal nitric oxide sunthase are less vulnerable to hypoxic-ischemic injury. Neurobiol Dis 1996;3:64-71.

19. Balligand JL, Ungureanu-Longrois D, Simmons WW, Pimental D, Malinski TA, Kapturczak M, Taha Z, Lowenstein CJ, Davidoff AJ, Kelly RA, et al. Cytokine-inducible nitric oxide synthase (iNOS) expression in cardiac myocytes. Characterization and regulation of iNOS expression and detection of iNOS activity in single cardiac myocytes in vitro. J Biol Chem 1994;269:27580-8.

20. van den Tweel ER, Peeters-Scholte CM, van Bel F, Heijnen CJ, Groenendaal F. Inhibition of nNOS and iNOS following hypoxia-ischaemia improves long-term outcome but does not influence the inflammatory response in the neonatal rat brain. Dev Neurosci 2002;24:389-95.

21. Karra D, Dahm R. Transfection techniques for neuronal cells. J Neurosci 2010;30:6171-7.

22. Colin A, Faideau M, Dufour N, Auregan G, Hassig R, Andrieu T, Brouillet E, Hantraye P, Bonvento G, Deglon N. Engineered lentiviral vector targeting astrocytes in vivo. Glia 2009;57:667-79.

23. Kim JJ, Fanselow MS. Modality-specific retrograde amnesia of fear. Science 1992;256:675-7. 
24. van Asselen M, Kessels RP, Neggers SF, Kappelle LJ, Frijns CJ, Postma A. Brain areas involved in spatial working memory. Neuropsychologia 2006;44:1185-94

25. Kapinya KJ, Lowl D, Futterer C, Maurer M, Waschke K, Isaev NK, Dirnagl U. Tolerance Against Ischemic Neuronal Injury Can Be Induced by Volatile Anesthetics and Is Inducible NO Synthase Dependent. Stroke 2002;33:1889-98.

26. Luo CX, Zhu XI, Zhou QG, Wang B, Wang W, Cai HH, Sun YJ, Hu M, Jiang J, Hua Y, Han X, Zhu DY. Reduced neuronal nitric oxide synthase is involved in ischemia-induced hippocampal neurogenesis by up-regulating inducible nitric oxide synthase expression. J Neurochem 2007;103:1872-82.

27. Pacher P, Beckman JS, Liaudet L. Nitric oxide and peroxynitrite in health and disease. Physiol Rev 2007;87:315-424.

28. Stamler JS, Singel DJ, Loscalzo J. Biochemistry of nitric oxide and its redox-activated forms. Science 1992;258:1898-902

29. Gross SS, Wolin MS. Nitric oxide: pathophysiological mechanisms. Annu Rev Physiol 1995;57:737-69.
30. Ischiropoulos $\mathrm{H}$, Beckman JS Oxidative stress and nitration in neurodegeneration: cause, effect, or association? J Clin Invest 2003;111:163-9.

31. Lefer DJ, Scalia R, Campbell B, Nossuli T, Hayward R, Salamon M, Grayson J, Lefer AM. Peroxynitrite inhibits leukocyte-endothelial cell interactions and protects against ischemia-reperfusion injury in rats. J Clin Invest 1997;99:684-91.

32. Klotz LO, Schroeder P, Sies H. Peroxynitrite signaling: receptor tyrosine kinases and activation of stress-responsive pathways. Free Radic Biol Med 2002;33:737-43.

33. Zhu DY, Liu SH, Sun HS, Lu YM. Expression of inducible nitric oxide synthase after focal cerebral ischemia stimulates neurogenesis in the adult rodent dentate gyrus. J Neurosci 2003;23:223-9.

34. Hierholzer C, Kalff JC, Billiar TR, Bauer AJ, Tweardy DJ, Harbrecht BG. Induced nitric oxide promotes intestinal inflammation following hemorrhagic shock. Am J Physiol Gastrointest Liver Physiol 2004;286:G225-33. 